\title{
CYCLIC VECTORS OF INDUCED REPRESENTATIONS
}

\section{A. HULANICKI AND T. PYTLIK}

In this note we prove that for a first countable locally compact group every unitary representation induced by a cyclic representation is cyclic.

This result has been recently obtained also by $F$. Greenleaf and M. Moskowitz [2] in a more complicated way.

Let $G$ be a first countable locally compact group. Let $\mathscr{K}$ be the space of continuous functions with compact support equipped with the Schwartz topology on $G$. Let $D$ be the cone in $\mathscr{K}^{\prime}$ of positive-definite measures on $G$, i.e., $\mu \in D$ if $\left\langle x^{*} * x, \mu\right\rangle \geqq 0$ for all $x$ in $\mathscr{K}$. For each $\mu$ in $D$ we define $I=\left\{x \in \mathscr{K}:\left\langle x^{*} * x, \mu\right\rangle=0\right\}$. Then $\mathscr{H}_{\mu}^{0}=\mathscr{K} \mid I$ is a pre-Hilbert space with a strictly positive-definite inner product $(\bar{x}, \tilde{y})_{\mu}=\left\langle y^{*} * x, \mu\right\rangle$, where $x \rightarrow \bar{x}$ is the natural mapping of $\mathscr{K}$ onto $\mathscr{H}_{\mu}^{0}$. Moreover, if $L_{g} x(h)=$ $x\left(g^{-1} h\right), g, h \in G$, then $I$ is stable under $L_{g}, g \in G$, and so $L_{g}$ acts on $\mathscr{H}_{\mu}^{0}$ and is unitary with respect to $(\cdot, \cdot)_{\mu}$. As such it extends to the completion $\mathscr{H}_{\mu}$ of $\mathscr{H}_{\mu}^{0}$. Let $g \rightarrow L_{g}^{\mu}$ be the representation thus obtained. If $P$ is a projection in $\mathscr{H}_{\mu}$ which commutes with the $L_{g}^{\mu}, g \in G$, then there exists a unique measure $v$ in $D$ such that

$$
(P \bar{x}, \bar{y})_{\mu}=\left\langle y^{*} * x, v\right\rangle \text { for all } x, y \text { in } \mathscr{K} .
$$

For the details and proofs cf. [1, pp. 40-48].

Let $\left\{e_{n}\right\}, n=1,2,3, \cdots$, be an approximate unit in $\mathscr{K}$ and let

$$
\xi=\sum_{1}^{\infty} \lambda_{n} e_{n}^{*} * e_{n},
$$

where the $\lambda_{n}$ are positive and such that the series is uniformly convergent.

THEOREM. For every measure $\mu$ in $D$, the vector $\bar{\xi}$ is cyclic with respect to $L_{g}^{\mu}$ in $\mathscr{H}_{\mu}$.

Proof. Suppose that $\bar{\xi}$ belongs to a subspace $\mathscr{N}$ of $\mathscr{H}_{\mu}$ which is $L_{g}^{\mu}$ invariant, $g \in G$. Let $P$ be a projection onto $\mathscr{N} \perp$. Clearly, $P$ commutes with $L_{g}^{\mu}$ and so it defines a measure $\nu$ in $D$ such that $(*)$ holds. Since

Received by the editors March 26, 1971.

AMS 1969 subject classifications. Primary 2260.

(c) American Mathematical Society 1972 
$P \bar{\xi}=0$, for every $e_{n}, n=1,2,3, \cdots$, we have $0=\left(P \bar{\xi}, \bar{e}_{n}\right)=\left\langle e_{n}^{*} * \xi, v\right\rangle$, whence

$$
\langle\xi, v\rangle=0 \text { and } \sum_{1}^{\infty} \lambda_{n}\left(e_{n}^{*} * e_{n}, v\right)=0 .
$$

Thus, since $\lambda_{n}>0$ and $\left\langle e_{n}^{*} * e_{n}, v\right\rangle \geqq 0$, we have $\left\langle e_{n}^{*} * e_{n}, v\right\rangle=0$ for all $n=1,2,3, \cdots$, which, by Schwartz inequality, means that $\left\langle e_{n} * x, v\right\rangle=0$ for all $x$ in $\mathscr{K}$ and $n=1,2,3, \cdots$, so $\nu=0$, which implies that $P=0$ and $\mathscr{N}=\mathscr{H}_{\mu}$.

Since every unitary representation induced from a cyclic representation is of the form $g \rightarrow L_{g}^{\mu}$ for a positive-definite measure $\mu$ (cf. [1]) we have

COROLlaRY. For a first countable locally compact group every unitary representation induced by a cyclic representation is cyclic.

\section{REFERENCES}

1. E. Effros and F. Hahn, Locally compact transformation groups and $C^{*}$-algebras, Mem. Amer. Math. Soc. No. 75 (1967). MR 37 \#2895.

2. F. Greenleaf and M. Moskowitz, Cyclic vectors for representations of locally compact groups (to appear).

Institute of Mathematics, The Polish Academy of Sciences, Warsaw, Poland Institute of Mathematics, University of Wroceaw, Wroceaw, Poland 